\title{
Prevention of the Postthrombotic Syndrome with Anticoagulation: A Narrative Review
}

\author{
Ilia Makedonov ${ }^{1}$ Susan R. Kahn ${ }^{2}$ Jameel Abdulrehman ${ }^{3}$ Sam Schulman ${ }^{40}$ Aurelien Delluc $5,6 \odot$ \\ Peter Gross ${ }^{7}$ Jean-Philippe Galanaud ${ }^{8}$
}

${ }^{1}$ Department of Medicine, Sunnybrook Health Sciences Center, Toronto, Ontario, Canada

2 Department of Medicine and Lady Davis Institute, Jewish General Hospital, Montreal, Quebec, Canada

${ }^{3}$ Division of Hematology, Department of Medicine, University Health Network, University of Toronto, Toronto, Ontario, Canada

${ }^{4}$ Thrombosis and Atherosclerosis Research Institute, Department of Medicine, McMaster University, Hamilton, Ontario, Canada

${ }^{5}$ Ottawa Hospital Research Institute and University of Ottawa, Ottawa, Ontario, Canada

${ }^{6}$ Division of Hematology, Department of Medicine, The Ottawa Hospital and University of Ottawa, Ottawa, Ontario, Canada

${ }^{7}$ Department of Medicine, McMaster University and the Thrombosis and Atherosclerosis Research Institute, Hamilton, Ontario, Canada

${ }^{8}$ Department of Medicine, Sunnybrook Health Sciences Centre and University of Toronto, Toronto, Ontario, Canada

\begin{abstract}
Address for correspondence Jean-Philippe Galanaud, MD, PHD, Department of Medicine, Sunnybrook Health Sciences Centre, 2075 Bayview Avenue Room D 675 B, Toronto, Ontario CAN M4N 3M5, Canada (e-mail: Jean-Philippe.Galanaud@sunnybrook.ca).
\end{abstract}

\section{Abstract}

Keywords

- postthrombotic syndrome

- venous thrombosis

- low molecular weight heparin

- vitamin K-dependent factors

- direct oral anticoagulants
The postthrombotic syndrome (PTS) is chronic venous insufficiency secondary to a prior deep vein thrombosis (DVT). It is the most common complication of venous thromboembolism (VTE) and, while not fatal, it can lead to chronic, unremitting symptoms as well as societal and economic consequences. The cornerstone of PTS treatment lies in its prevention after DVT. Specific PTS preventative measures include the use of elastic compression stockings and pharmacomechanical catheter-directed thrombolysis. However, the efficacy of these treatments has been questioned by large randomized controlled trials (RCTs). So far, anticoagulation, primarily prescribed to prevent DVT extension and recurrence, appears to be the only unquestionably effective treatment for the prevention of PTS. In this literature review we present pathophysiological, biological, radiological, and clinical data supporting the efficacy of anticoagulants to prevent PTS and the possible differential efficacy among available classes of anticoagulants (vitamin K antagonists [VKAs], low molecular weight heparins [LMWHs] and direct oral anticoagulants [DOACs]). Data suggest that LMWHs and DOACs are superior to VKAs, but no head-to-head comparison is available between DOACs and LMWHs. Owing to their potentially greater anti-inflammatory properties, LMWHs could be superior to DOACs. This finding may be of interest particularly in patients with extensive DVT at high risk of moderate to severe PTS, but needs to be confirmed by a dedicated RCT. received

July 13,2021

accepted after revision

November 24, 2021

published online

February 15, 2022 (c) 2022. Thieme. All rights reserved. Georg Thieme Verlag KG,

Rüdigerstraße 14,

70469 Stuttgart, Germany
DOI https://doi.org/

10.1055/a-1711-1263.

ISSN 0340-6245. 


\section{Introduction}

The postthrombotic syndrome (PTS) is chronic venous insufficiency (CVI) secondary to a prior deep vein thrombosis (DVT). ${ }^{1}$ PTS is the most common long-term complication of venous thromboembolism (VTE), developing in 20 to $50 \%$ of patients after a proximal DVT. While PTS is not lethal, it is an important public health issue as it has serious medical and economic consequences. ${ }^{2}$ It is a strong predictor of impaired quality of life and increased cost after a DVT. ${ }^{3}$ Indeed, PTS can lead to daily, nonremitting symptoms and, in severe cases, to venous ulcers. Patients with PTS report worse quality of life scores than patients with chronic diseases such as arthritis, chronic lung disease, and diabetes, ${ }^{4,5}$ whereas those with severe PTS can have a quality of life similar to patients with malignancy, angina, and congestive heart failure. ${ }^{5}$

From a health economics point of view, PTS increases the cost of DVT treatment by 35 to $45 \%$ compared with uncomplicated DVT. ${ }^{2,3,6}$ Before 2014, in the absence of welltolerated and effective treatments for established PTS, the cornerstone of PTS management was prevention ${ }^{7}$ with daily use of elastic compression stockings (ECS) for 2 years and, in selected cases, with catheter-directed thrombolysis (CDT) to treat acute DVT. More recently, after the SOX trial failed to show a benefit from ECS ${ }^{8}$ and the Acute Venous Thrombosis: Thrombus Removal with Adjunctive Catheter-Directed Thrombolysis (ATTRACT) trial failed to show a benefit from $\mathrm{CDT},{ }^{9}$ the efficacy of these treatments has been questioned, ${ }^{9,10}$ and some doctors may be feeling "empty handed." ${ }^{11}$ However, even if specific PTS preventative measures are ineffective or less effective than previously believed, it should be kept in mind that there exists a simple, effective, but nonspecific measure to prevent PTS: anticoagulant treatment. ${ }^{12}$ This is prescribed to all proximal DVT patients, primarily to prevent DVT extension, pulmonary embolism (PE), and VTE recurrence, but is also effective for preventing PTS. In this review, we will discuss the evidence supporting the effectiveness of anticoagulation for the prevention of PTS as well as associated mechanisms. We will also review whether the choice of anticoagulant may influence the risk of developing PTS and whether some anticoagulants could be favored over others with respect to PTS prevention.

\section{Methods}

We conducted a pubmed.gov and clinicaltrials.gov registry search (from inception up until December 8, 2020) to identify studies evaluating the effect of vitamin $\mathrm{K}$ antagonists (VKAs), low molecular weight heparins (LMWHs), and direct oral anticoagulants (DOACs) on PTS and comparing one anticoagulant to another with respect to PTS. The full search strategy is in -Supplementary Appendix 1 (available in the online version). Only English, French, and Russian language literature was considered. References from all relevant papers were reviewed.

\section{Effectiveness of Anticoagulation for Preventing PTS after DVT}

Heparin (either unfractionated or, later, low molecular weight) for 7 to 10 days followed by a VKA was the standard of care for the treatment of VTE from the 1940s until the development of DOACs in the 21 st century. ${ }^{13}$ A retrospective historical series from 1946 showed that anticoagulation reduced the signs and symptoms of PTS by a significant degree compared with no treatment. The presence of any swelling was reduced by $79 \%$, induration and ulceration were both reduced by $100 \%$, and the risk of heaviness decreased by $95 \% .{ }^{14}$ While this was not a controlled study, it is suggestive of a profound effect of anticoagulation on the risk of PTS. Modern data also support the importance of therapeutic anticoagulation for the prevention of PTS. Patients who spend more than $50 \%$ of time beneath an international normalized ratio (INR) of 2.0 have a 2.71 odds ratio (OR) of developing PTS (1.44-5.1). ${ }^{15}$ Another study found that the OR of developing PTS was 1.94 (1.133.01 ) in patients with subtherapeutic INR values within the first 3 months of treatment. ${ }^{16}$ Although we would no longer be able to randomize anticoagulation against placebo in an acute proximal DVT treatment trial, a recent randomized controlled trial (RCT) included 178 patients with distal DVT and randomized them to either therapeutic LMWH (nadroparin) or placebo for 6 weeks. ${ }^{17}$ It showed that in the subgroup of patients without evidence of primary CVI, the rate of PTS at 6 years might be lower when treated with LMWH ( $9 \%$ vs. $24 \%, p=0.04$ ); however, this was a subgroup analysis. Current guidelines endorse good-quality anticoagulation as an effective tool for prevention of PTS but do not suggest a specific anticoagulant agent. ${ }^{12}$ Regarding the duration of anticoagulant treatment, there does not appear to be a benefit from extending anticoagulant treatment beyond the usual treatment duration with respect to PTS prevention. Thus, in the ExACT trial that randomized 281 patients with proximal VTE to receive either 3 months or 2 years of anticoagulation, there was no difference in terms of Villalta score or venous quality of life scores between groups at 2 years. ${ }^{18}$ Similarly, in the DURAC trial there was no difference in the rates of PTS at 10 years between patients treated with 6 weeks or 6 months of anticoagulation. ${ }^{19}$ In summary, both historic and modern data suggest that anticoagulation and quality of anticoagulation are critical at the acute phase of DVT for preventing PTS. This raises the question of the pathophysiological mechanism that could explain why anticoagulation prevents PTS after DVT.

\section{Anticoagulation and PTS Pathophysiology}

There is rapid thrombus regression during the first 2 to 3 months after the onset of anticoagulant treatment for acute DVT and, after 3 months, thrombus regression is gradual and slow. ${ }^{20}$ After 2 years, no additional thrombus regression is expected and the degree of residual venous obstruction (RVO) is fixed. From a hemodynamic point of 
view, venous valvular reflux follows thrombus resolution and early thrombus resolution is associated with better valve preservation and reduced reflux. ${ }^{20}$ Furthermore, the smaller the clot burden, the lower the risks of RVO, venous reflux, and ultimately PTS..$^{12}$ This is the rationale for the use of CDT in extensive DVT, ${ }^{21}$ but any treatment that is able to reduce the initial clot burden should reduce the risk of PTS. This is likely why all anticoagulation is effective for prevention of PTS.

In addition to RVO and venous reflux, there is also a significant inflammatory component to thrombus formation that is felt to contribute to $\mathrm{PTS}^{22}$ ( - Fig. 1). The formation of a thrombus involves increased release of inflammatory cytokines (tissue necrosis factor- $\alpha$ [TNF- $\alpha$ ], interleukin [IL]-6, IL8 ), increased expression of adhesion molecules (P- and Eselectins, intercellular adhesion molecule 1 [ICAM-1], vascular cell adhesion molecule 1 [VCAM-1]), platelet and leukocyte adhesion, leukocyte migration into the vessel wall, and formation of neutrophil extracellular traps. ${ }^{23-26}$ From a hemodynamic point of view, higher levels of some inflammation markers have been associated with a reduced probability of venous recanalization (IL-6 and P-selectin) $)^{27}$ and with increased venous outflow resistance (IL-6). ${ }^{28}$ This translates clinically to a higher risk of PTS. Thus, in a Canadian study, elevated IL-6 levels were associated with an increased risk of PTS (OR 1.66 [1.05-2.62]). ${ }^{29}$ Similarly, the Bio-SOX study found that elevated levels of ICAM- 1 and IL-10 were also predictive of an increased risk of PTS (relative risk [RR] 1.25 [1.05-1.48] and 1.27 [1.07-1.51], respectively). ${ }^{22}$ Importantly, there was a dose response between ICAM-
1 level and the risk of PTS, providing support for a key role of inflammation in PTS development. ${ }^{22}$ In the same line, matrix metalloproteinases (MMPs), which are enzymes that regulate inflammatory mediators and maintain the integrity of physical barriers, ${ }^{23}$ have also be found to be associated with persistent thrombosis (MMP-9) ${ }^{30}$ and PTS (MMP-1 and MMP-8). ${ }^{31}$ In corroboration, animal models also showed that inflammation delayed thrombus resolution ${ }^{23,30}$ and promoted vein wall injury. ${ }^{32}$ Thus, therapies that reduce inflammation have the potential to improve thrombus resolution, vein wall remodeling, and to thereby prevent PTS. There is ongoing research to identify associated pathways, including in the pediatric population. ${ }^{33}$

The inflammatory response is most strongly pronounced during the first month after acute VTE. The Bio-SOX study showed that inflammatory markers (C-reactive protein, IL-6) rapidly decreased during the first month after diagnosis, and only marginally decreased over the five subsequent months. ${ }^{22}$ Additionally, baseline inflammatory marker elevation predicted subsequent PTS. ${ }^{22}$ This suggests that an anticoagulant with potent anti-inflammatory properties would provide the most benefit during the first month of treatment (-Fig. 1).

By reducing clot extension, anticoagulants may prevent RVO and venous reflux, two important components of PTS physiopathology. Anticoagulants may to some degree also influence the third component of PTS pathophysiology: inflammation. The differential impact on inflammation of the various types of anticoagulants might translate to

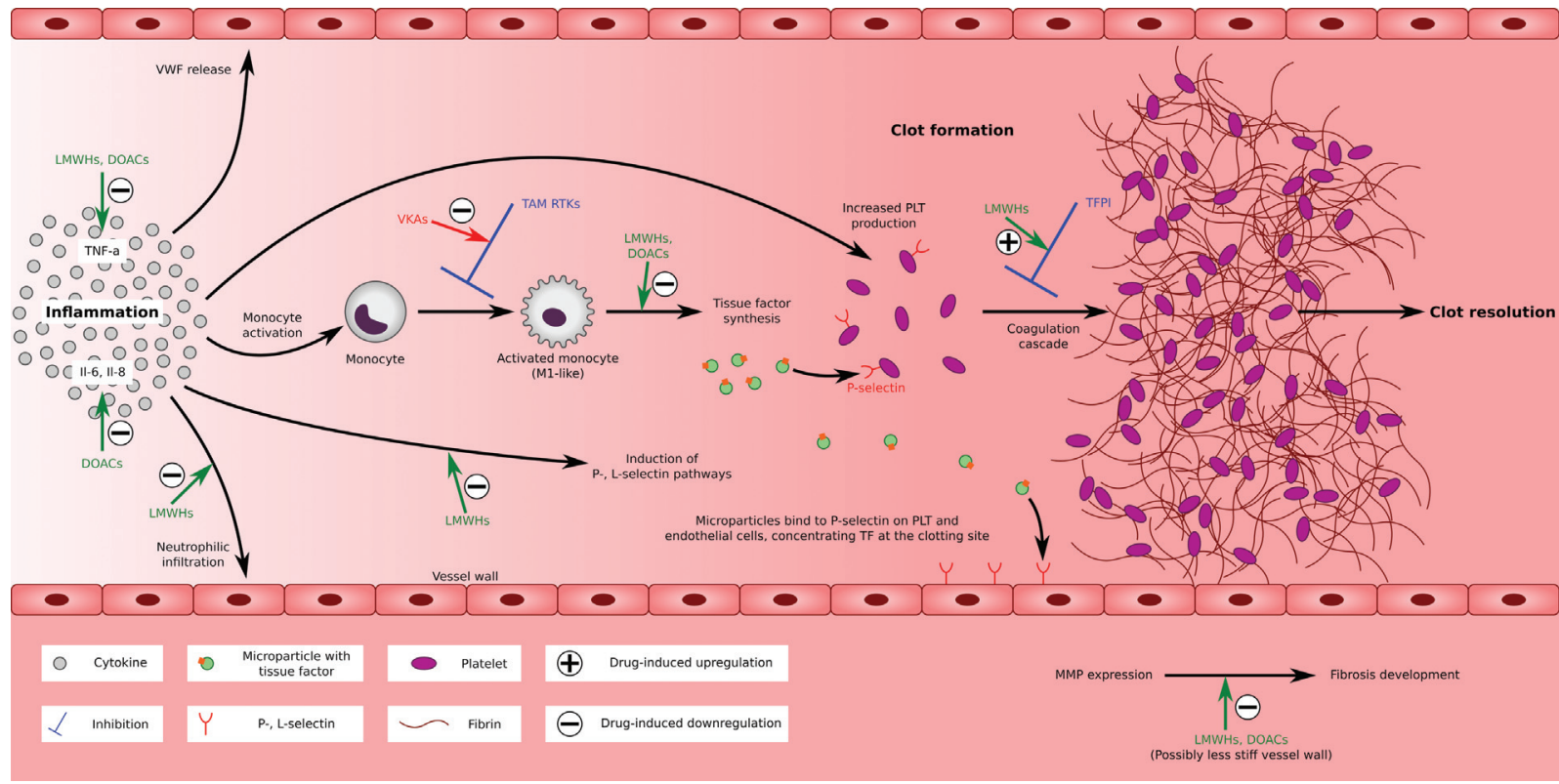

Fig. 1 Inflammatory cytokines are predisposing factors for VTE (e.g., IL6, IL 8, TNF- $\alpha$ ) and can activate monocytes, induce P- and L-selectin pathways, promote release of VWF, increase platelet production, and promote neutrophilic infiltration. TAM family receptor tyrosine kinases (TAM RTKs) inhibit inflammatory signaling, and are found on the surface of monocytes, macrophages, and other cells. Activated monocytes propagate the coagulation cascade through tissue factor synthesis and production of microparticles. These in turn adhere to selectin receptors found on both platelets and endothelial cells. MMPs affect thrombus resolution and collagen formation. MMP-9 may increase the stiffness of remodeled venous walls. VKAs may promote coagulation through their inhibition of the TAM RTKs. TNF- $\alpha$ levels are decreased by both LMWHs and DOACs. IL-6 and IL-8 levels are reduced by DOACs. Neutrophilic infiltration and selectin pathways are inhibited by LMWHs. TF synthesis is reduced by LMWHs and DOACs. MMP-9 levels are reduced by both LMWHs and DOACs. DOAC, direct oral anticoagulant; IL6, interleukin 6; IL 8, interleukin 8; LMWH, low molecular weight heparin; MMP, matrix metalloproteinase; TAM, Tyro3, Axl and Mer; TNF- $\alpha$, tumor necrosis factor $\alpha$; VKA, vitamin $\mathrm{K}$ antagonist; VTE, venous thromboembolism; VWF, von Willebrand factor. 
differential effectiveness in PTS prevention. In the next section, we will review, for each class of anticoagulant (VKAs, LMWHs, and DOACs), their impact on inflammation as well as the radiologic and clinical evidence supporting their variable efficacy for prevention of PTS.

\section{Vitamin K Antagonists}

VKAs reduce the amount of active vitamin $\mathrm{K}$ available for the synthesis of vitamin K-dependent clotting factors. ${ }^{34}$ Vitamin K is also used in pathways involving the TAM receptor tyrosine kinases (RTKs), which are distinct from the coagulation cascade. VKAs may influence inflammation by inhibiting growtharrest-specific 6, which activates several RTKs (MERTK and AXL). ${ }^{35}$ MERTK and AXL inhibit the innate immune system, including monocyte activity. ${ }^{36}$ Monocytes play an important proinflammatory role in acute VTE, preventing thrombus maturation and resolution. ${ }^{25}$ Therefore, from a theoretical point of view, by reducing the activation of RTKs, VKA may reduce fibrinolysis and collagenolysis. Murine models have shown increased systemic and local levels of inflammatory markers such as IL-6, IL-17, and interferon- $\gamma$ after exposure to VKAs, ${ }^{37,38}$ although the results have not been consistent. ${ }^{39} \mathrm{~A}$ recent literature review suggests that VKAs are favored to be proinflammatory. ${ }^{35}$ Anticoagulants that do not possess such proinflammatory properties or those that have anti-inflammatory properties are likely to be potentially more effective than VKAs with respect to PTS prevention.

\section{Low Molecular Weight Heparins}

\section{Impact of LMWHs on Inflammation}

LMWHs are linear polysaccharide structures, and have been used clinically since the 1980 s. $^{13}$ They are derivatives of unfractionated heparin (UFH), which originates from mast cells found predominantly in porcine and bovine intestinal mucosa. Both heparin and LMWHs have strong anti-inflammatory properties, owing to their glycan chains. ${ }^{40-45}$ LMWHs release tissue factor pathway inhibitor (TFPI), ${ }^{46}$ block P-selectin interactions, ${ }^{47}$ and inhibit lymphocytes, chemokines, fibroblast proliferation, ${ }^{43,47,48}$ endothelial activation, and neoangiogenesis. ${ }^{44,49-51}$ Experimental rodent models of DVT have reported that LMWH reduced the size of the clot and inflammatory cell extravasation into the vein compared with controls, ${ }^{43}$ and was found to have a protective effect on the vein wall by promoting re-endothelialization $^{42}$ and reducing fibrosis ${ }^{52}$ and intimal hyperplasia. ${ }^{53}$ Importantly, there was a dose-response effect, with greater benefits with higher doses of LMWH, suggesting a true biological effect. ${ }^{43}$ As compared with VKA, in a RCT of 1,048 patients with acute DVT, treatment with LMWH (reviparin) significantly reduced the inflammatory response at 21 days, as reflected by higher levels of TFPI, and lower levels of fibrinogen and of thrombin activatable fibrinolysis inhibitor. ${ }^{54}$ These laboratory data suggest that, unlike VKAs, LMWHs have strong anti-inflammatory properties which could theoretically lead to better effectiveness in PTS prevention.

\section{Radiologic Evidence of LMWH versus VKA Effectiveness for PTS Prevention}

Radiologic data suggests that LMWH use results in higher recanalization rates than VKA use. There have been six randomized trials that compared VKAs to various doses of LMWHs and assessed radiologic outcomes. ${ }^{55-60}$ Three of these used prophylactic dosing ${ }^{55-57}$ and the other three used therapeutic dosing ${ }^{58-60}$; patient numbers ranged from 105 to 324, and LMWH treatment duration ranged between 3 and 6 months. The trials that used prophylactic dosing for extended treatment all had an upfront period of 7 to 10 days of either UFH or full dosing LMWH. Five of the six trials found either more complete or more rapid recanalization in the LMWH arm, and one meta-analysis of 1,006 patients also found improved recanalization with $\mathrm{LMWH}$ $(\mathrm{RR}=0.49$ [0.26-0.92] and $\mathrm{RR}=0.73$ [0.53-1.01] for therapeutic and prophylactic dosing, respectively). ${ }^{56}$ However, heterogeneity across studies was significant, reducing the quality of the data. Interestingly, the improved recanalization rate was not associated with reduced rates of VTE recurrence, suggesting that mechanisms other than VTE recurrence accounted for the protective effect. ${ }^{56}$

\section{Clinical Evidence of LMWH versus VKA Effectiveness for PTS Prevention}

There have been four randomized trials comparing LMWHs to VKAs with respect to PTS prevention, with one using prophylactic dosing, ${ }^{61}$ and the other three using therapeutic dosing. ${ }^{59,60,62}$ Patient numbers ranged between 100 and 480, and treatment duration with LMWH was between 3 and 6 months. One of the trials used the Villalta scale to quantify PTS, ${ }^{61}$ two trials used nonvalidated clinical observations, ${ }^{59,60}$ and the fourth trial used a self-reported patient scale. $^{62}$ The three smaller trials favored LMWH in terms of lower PTS rates, but results did not reach statistical significance..$^{59-61}$ The largest trial reported a statistically significant reduction in PTS with LMWH at 12 weeks $(\mathrm{OR}=0.77$ [0.66-0.91], $p=0.001) .{ }^{62}$ This was a multicenter Canadian trial that randomized patients to 3 months of tinzaparin or a VKA, and included 480 patients. The presence of PTS was evaluated at 12 weeks, and the presence of ulcers was evaluated at 12 weeks and 1 year. Unfortunately, the proportion of iliac and common femoral vein DVTs and the extent of INR control were not documented. A meta-analysis was not able to combine PTS outcomes due to the variable follow-up periods ( 3 months -5 years) and variable assessment methods, but it did compare the risk of venous ulcers. Based on two studies, the risk of venous ulcers, which represent the most severe form of PTS, was significantly and strongly reduced with LMWH $(\mathrm{RR}=0.31$ [0.02-0.71], $p=0.019) .{ }^{63}$ See - Table 1 for a summary of the studies.

\section{Summary}

Unlike VKAs, LMWHs have strong anti-inflammatory properties. Animal models have shown a favorable effect of LMWHs on the natural history of thrombosis, with more rapid resolution, faster re-endothelialization, and reduced fibrosis. Radiologic data in human patients have shown 


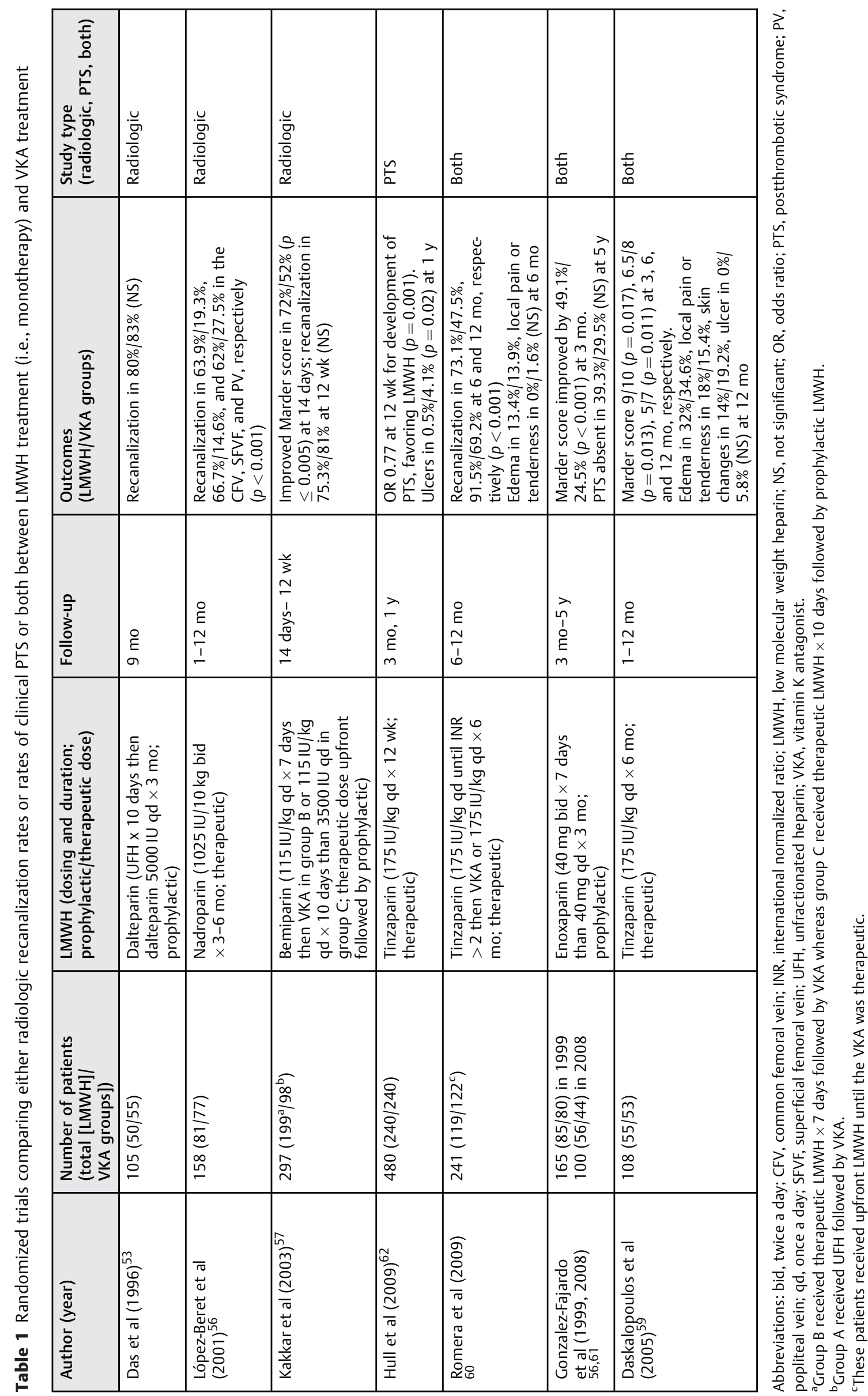


improved recanalization rates with LMWHs compared with VKAs, independent of VTE recurrence rates. While there are few RCTs, they do report a significant reduction in PTS risk after extended treatment of DVT with LMWH. The mechanisms for this may relate to nonanticoagulant properties of LMWHs.

\section{Direct Oral Anticoagulants}

\section{Impact of DOAC on Inflammation}

DOACs are synthetic molecules that inhibit factors IIa or Xa. Laboratory evidence suggests that they also target inflammatory pathways. Murine data shows that rivaroxaban decreases infarct size in a model of myocardial infarction ${ }^{64}$ and reduces the release of inflammatory markers (IL-6, TNF- $\alpha$, MMP-9) in models of atherosclerosis. ${ }^{65,66}$ Human vascular endothelial cell models of oxidative damage have shown an increased release of IL-10, a potent anti-inflammatory cytokine, ${ }^{67}$ and reduced expression of inflammatory genes (VCAM-1, ICAM- 1 , IL-8, TF). ${ }^{68}$ A human monocyte model showed reduced release of inflammatory cytokines (IL-6) and chemokines (IL-8, monocyte chemoattractant protein-1) from cells activated by thrombin. ${ }^{69}$ A human plasma proteomics study showed that rivaroxaban increased thrombomodulin levels and showed a trend to decreased MMP-9 levels. ${ }^{70}$ However, there is an absence of studies that used DVT models, and most of the data originates from cell lines. Therefore, the relevance of these findings to PTS is less clear than it is for LMWHs.

\section{Radiologic Evidence of DOAC versus VKA Effectiveness for PTS Prevention}

Four retrospective studies ${ }^{71-74}$ and one $\mathrm{RCT}^{75}$ have compared vein recanalization rates in patients treated with DOACs and VKAs. Most of these studies investigated rivaroxaban. All five studies showed reduced rates of RVO with DOAC treatment, but one study did not reach statistical significance. ${ }^{73}$ Most studies reported outcomes at 3 to 12 months after index DVT, but Ferreira et al evaluated patients much later after the index $\mathrm{VTE}^{72}$; in this study, DOAC patients were evaluated at 15 months and VKA patients were evaluated at 61 months. This would be expected to favor the DOAC group, as the rate of PTS increases with time. Most studies reported on 77 to 129 patients, with Prandoni et al reporting on a larger retrospective group of 1,345 patients. The RCT by de Athayde Soares et al is unique in that it randomized patients to DOAC or VKA primarily to evaluate the effect on PTS. ${ }^{75}$ This Brazilian trial included 84 patients who were followed for a median of 1 year. The rate of complete recanalization at 1 year was higher in the DOAC arm versus VKA arm $(76.1 \%$ vs. $13.2 \%, p<0.01)$. Existing radiologic evidence supports the superiority of DOACs to VKAs with respect to recanalization rates.

\section{Clinical Evidence of DOAC versus VKA Effectiveness for PTS Prevention}

There have been four retrospective studies, ${ }^{72,76-78}$ two cross-sectional follow-up studies of the phase III DOAC trials, ${ }^{79,80}$ two registry-based studies, ${ }^{81,82}$ and one recent $\mathrm{RCT}^{75}$ comparing DOACs to VKAs for PTS. A recent metaanalysis included all of the above studies except for the RECOVER follow-up study (the phase III study of dabigatran compared with warfarin) and the registry-based studies. ${ }^{83}$ The retrospective studies ranged in size from 100 to 1,300 patients, and the registry studies included 20,000 to 37,000 patients. The DOAC employed in all follow-up studies was rivaroxaban, except for the RE-COVER follow-up study, which evaluated dabigatran. All the rivaroxaban studies reported a lower risk of PTS with rivaroxaban compared with VKA. The RE-COVER follow-up study did not show a difference between dabigatran and VKA in regards to PTS risk, ${ }^{80}$ and this study also employed the patient self-reported Villalta scale (while all other studies used the patient- and clinician-rated Villalta scale). The meta-analysis showed a protective effect of rivaroxaban compared with VKA on risk of PTS (adjusted OR $=0.44 \quad[0.35-0.56] ; \quad I^{2}=0$ with $p=0.42) .{ }^{83}$ The RE-COVER follow-up study showed overall higher rates of PTS, with $61 \%$ of patients in the DVT group and $44 \%$ of the patients in the PE-only group diagnosed with PTS at a mean follow-up of 8.7 years. ${ }^{80}$ These findings suggest that treatment-related effects may have been masked by naturally progressive baseline CVI, as PTS observed in patients in the PE-only group was more likely to be attributed to underlying CVI. Among registry studies, Coleman et al found a reduced risk of PTS with rivaroxaban treatment $(23 \%$ relative reduction [95\% confidence interval $16-30]$ ], ${ }^{81}$ and Søgaard et al found a nonsignificant trend to reduced PTS with rivaroxaban $(\mathrm{HR}=0.88[0.66-1.17]) .^{82}$ However, registry studies are based on diagnostic codes, and it is difficult to accurately capture a diagnosis as nuanced as PTS based on coding. The RCT by de Athayde Soares et al is unique in having randomized patients to DOAC versus VKA to specifically assess effect on PTS. Eighty-four patients were randomized to rivaroxaban versus VKA for 6 months, and the median follow-up time was 360 days. The study showed a lower risk of PTS with rivaroxaban than VKA at 360 days (8.7\% vs. $28.9 \%$, $p<0.001){ }^{75}$ However, there were significantly more patients with proximal DVT in the VKA group (23.9\% vs. $5.3 \%$ iliofemoral, $p=0.018$ ), which may have favored the rivaroxaban group. In the VKA group, there was good adherence to INR monitoring, with $95 \%$ of patients in the therapeutic range, and a median INR of 2.4. This suggests that the protective effect of rivaroxaban was not due to higher quality anticoagulation than the VKA group. This was a single-center trial, and the results require confirmation. Further, the authors' reporting of time in therapeutic range was not conventional, and was not clearly defined. Several observational studies are ongoing regarding the real-world incidence of PTS with DOACs, including the Italian Monitoring Anticoagulant Therapy Observational Study (MAC Project), which aims to recruit up to 4,000 Italian VTE patients. ${ }^{84}$ Current data supports the superiority of DOACs over VKAs for prevention of PTS, although among DOACs, rivaroxaban has been nearly exclusively studied, and data on other DOACs is lacking. 


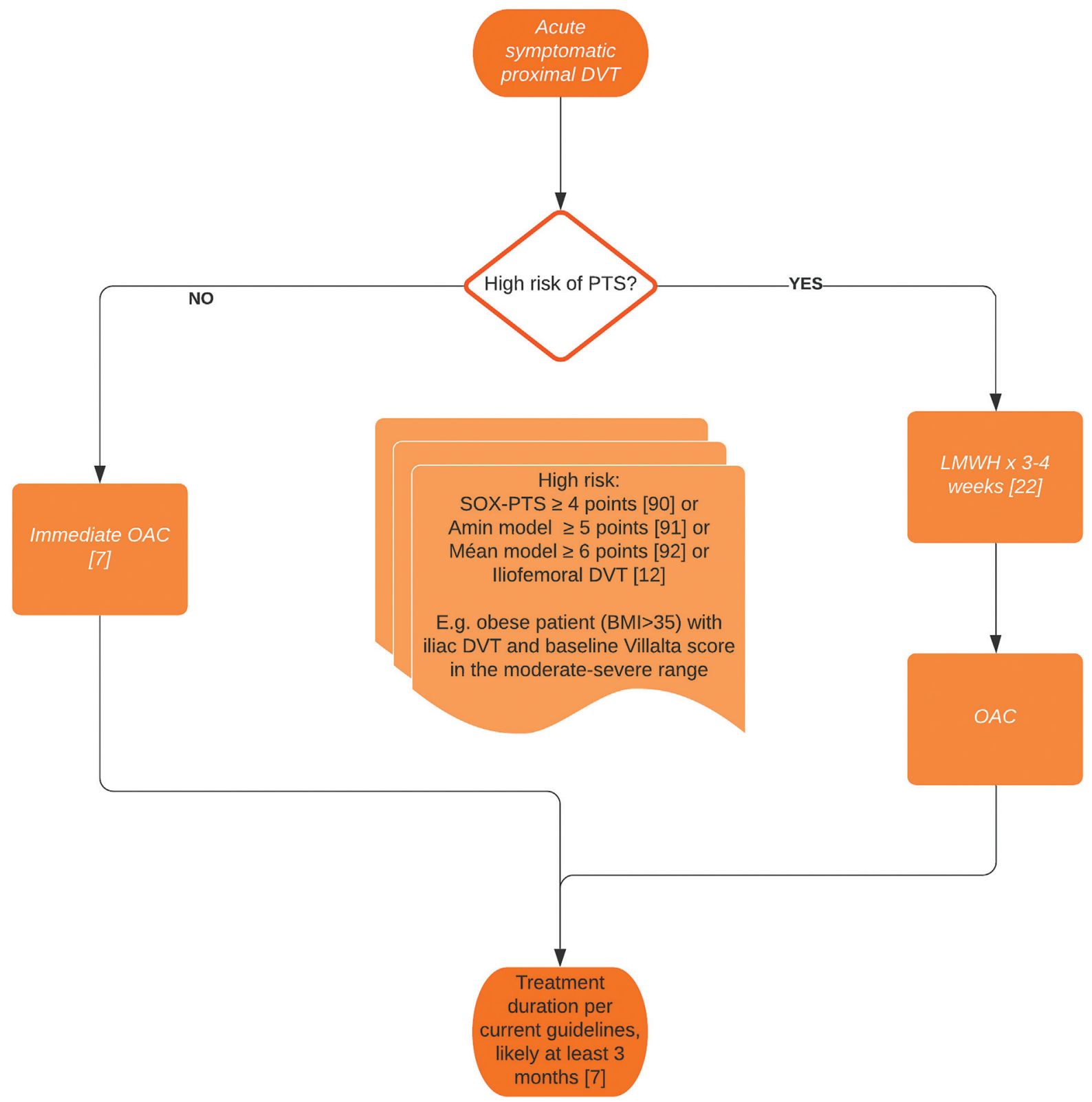

Fig. 2 Hypothetical management pathway for proximal deep vein thrombosis (DVT). The SOX-PTS, ${ }^{89}$ Amin, ${ }^{90}$ and Méan ${ }^{91}$ models predict the risk of PTS. Iliofemoral DVT has also been associated with an increased risk of PTS (odds ratio [OR] 6.3 [2.0-19.8]). ${ }^{12}$ Risk is calculated in the models as follows: SOX-PTS model: 0 points $-6.4 \%, 1$ point $-13.4 \%, 2$ points $-16.4 \%, 3$ points $-25 \%, \geq 4$ points $-30 \%$ risk of PTS; Amin model: $0-2$ points $-10 \%, 3-4$ points $-20 \%, \geq 5$ points $-40 \%$ risk of PTS; Méan model: $0-3$ points $-24.4 \%, 4-5$ points $-38.4 \%, \geq 6$ points-80.7\%. Please refer to the original publications regarding assignment of points. BMI, body mass index; LMWH, low molecular weight heparin; OAC, oral anticoagulant; PTS, postthrombotic syndrome.

\section{Clinical Evidence of LMWH versus DOAC Effectiveness for PTS Prevention}

As presented above, both LMWHs and DOACs appear to be more effective than VKAs for preventing PTS. DOACs are the current standard of care for the treatment of most patients with acute DVT, but no trial to date has compared LMWH to DOAC therapy for the prevention of PTS. In vitro laboratory data suggest that LMWHs may be more potent at inhibiting thrombin generation, delaying clot formation, and reducing maximum clot firmness than DOACs. ${ }^{85}$ While this is not readily translatable to clinical outcomes, it suggests that LMWHs may reduce the thrombotic and inflammatory bur- den in the acute phase of DVT compared with DOACs. Several experts in the field have called for trials comparing the efficacy of LMWHs to DOACs for prevention of PTS. ${ }^{86-88}$ As outlined previously, the inflammatory response is greatest in the first month following VTE, and patients with greater inflammatory changes at baseline are more likely to develop PTS. See - Fig. 2 for a hypothetical management pathway that takes into account the possible benefits of upfront LMWH treatment; high-risk patients can be classified based on clinical experience and PTS prediction models. ${ }^{89-91} \mathrm{Fu}-$ ture trials comparing LMWH versus DOAC for PTS prevention should probably compare the benefit of a heparin-lead-in 
course of up to 4 weeks of a LMWH to upfront DOAC treatment. Such a design appears pragmatic as patients in the noncancer setting may be averse to committing to 3 to 6 months of an injectable formulation for uncertain benefit with respect to PTS.

\section{Conclusion}

Timely and effective anticoagulant treatment constitutes, so far, the best way to prevent PTS after an acute DVT. Data suggest that both LMWHs and DOACs could be superior to VKAs for the prevention of PTS. This improved efficacy could be driven by LMWH and DOAC anti-inflammatory properties. LMWHs appear to have more potent anti-inflammatory properties than DOACs but no head-to-head comparison exists with respect to PTS prevention. Such an assessment is desirable, particularly in patients with extensive DVT, where the inflammatory response and risk of PTS are the highest. The TILE study will try to answer that question (ClinicalTrials.gov NCT04794569).

\section{Conflict of Interest}

J.P.G. received consultant fees from Sanofi S.A. and Servier Laboratories; research grants from Leo Pharma, Bayer AG, and Bristol-Myers Squibb-Pfizer; and a travel grant from Innothéra. Other authors have no conflicts to declare.

\section{References}

1 Makedonov I, Kahn SR, Galanaud J-P. Prevention and management of the post-thrombotic syndrome. J Clin Med 2020;9(04):923

2 Kachroo S, Boyd D, Bookhart BK, et al. Quality of life and economic costs associated with postthrombotic syndrome. Am J Health Syst Pharm 2012;69(07):567-572

3 Guanella R, Ducruet T, Johri M, et al. Economic burden and cost determinants of deep vein thrombosis during 2 years following diagnosis: a prospective evaluation. J Thromb Haemost 2011;9 (12):2397-2405

4 Lubberts B, Paulino Pereira NR, Kabrhel C, Kuter DJ, DiGiovanni $\mathrm{CW}$. What is the effect of venous thromboembolism and related complications on patient reported health-related quality of life? A meta-analysis. Thromb Haemost 2016;116(03):417-431

5 Kahn SR, Shbaklo H, Lamping DL, et al. Determinants of healthrelated quality of life during the 2 years following deep vein thrombosis. J Thromb Haemost 2008;6(07):1105-1112

6 MacDougall DA, Feliu AL, Boccuzzi SJ, Lin J. Economic burden of deep-vein thrombosis, pulmonary embolism, and post-thrombotic syndrome. Am J Health Syst Pharm 2006;63(20, Suppl 6): S5-S15

7 Kearon C, Akl EA, Comerota AJ, et al. Antithrombotic therapy for VTE disease: Antithrombotic therapy and prevention of thrombosis, 9th ed: American College of Chest Physicians evidencebased clinical practice guidelines. Chest 2012;141:e419S-e496S

8 Kahn SR, Shapiro S, Wells PS, et al; SOX trial investigators. Compression stockings to prevent post-thrombotic syndrome: a randomised placebo-controlled trial. Lancet 2014;383 (9920):880-888

9 Vedantham S, Goldhaber SZ, Julian JA, et al; ATTRACT Trial Investigators. Pharmacomechanical catheter-directed thrombolysis for deep-vein thrombosis. N Engl J Med 2017;377(23): 2240-2252

10 Galanaud JP, Righini M, Quéré I Compression stockings to prevent post-thrombotic syndrome. Lancet 2014;384(9938):129
11 ten Cate-Hoek AJ. Elastic compression stockings-is there any benefit? Lancet 2014;383(9920):851-853

12 Kahn SR, Comerota AJ, Cushman M, et al; American Heart Association Council on Peripheral Vascular Disease, Council on Clinical Cardiology, and Council on Cardiovascular and Stroke Nursing. The postthrombotic syndrome: evidence-based prevention, diagnosis, and treatment strategies: a scientific statement from the American Heart Association. Circulation 2014;130(18): 1636-1661

13 Galanaud JP, Laroche JP, Righini M. The history and historical treatments of deep vein thrombosis. J Thromb Haemost 2013;11 (03):402-411

14 Bauer G. Thrombosis; early diagnosis and abortive treatment with heparin. Lancet 1946;1(6396):447-454

15 van Dongen CJJ, Prandoni P, Frulla M, Marchiori A, Prins MH, Hutten BA. Relation between quality of anticoagulant treatment and the development of the postthrombotic syndrome. J Thromb Haemost 2005;3(05):939-942

16 Chitsike RS, Rodger MA, Kovacs MJ, et al. Risk of post-thrombotic syndrome after subtherapeutic warfarin anticoagulation for a first unprovoked deep vein thrombosis: results from the REVERSE study. J Thromb Haemost 2012;10(10):2039-2044

17 Galanaud JP, Righini M, Le Collen L, et al. Long-term risk of postthrombotic syndrome after symptomatic distal deep vein thrombosis: the CACTUS-PTS study. J Thromb Haemost 2020;18 (04):857-864

18 Bradbury C, Fletcher K, Sun Y, et al. A randomised controlled trial of extended anticoagulation treatment versus standard treatment for the prevention of recurrent venous thromboembolism (VTE) and post-thrombotic syndrome in patients being treated for a first episode of unprovoked VTE (the ExACT study). Br J Haematol 2019:bjh.16275

19 Schulman S, Lindmarker P, Holmström M, et al. Post-thrombotic syndrome, recurrence, and death 10 years after the first episode of venous thromboembolism treated with warfarin for 6 weeks or 6 months. J Thromb Haemost 2006;4(04):734-742

20 van Rij AM, Hill G, Krysa J, et al. Prospective study of natural history of deep vein thrombosis: early predictors of poor late outcomes. Ann Vasc Surg 2013;27(07):924-931

21 Watson L, Broderick C, Armon MP. Thrombolysis for acute deep vein thrombosis (Review) summary of findings for the main comparison. Cochrane Database Syst Rev 2016:1-59

22 Rabinovich A, Cohen JM, Cushman M, et al. Inflammation markers and their trajectories after deep vein thrombosis in relation to risk of post-thrombotic syndrome. J Thromb Haemost 2015;13(03): 398-408

23 Wakefield TW, Myers DD, Henke PK. Mechanisms of venous thrombosis and resolution. Arterioscler Thromb Vasc Biol 2008; 28(03):387-391

24 Mosevoll KA, Johansen S, Wendelbo Ø, Nepstad I, Bruserud Ø, Reikvam H. Cytokines, adhesion molecules, and matrix metalloproteases as predisposing, diagnostic, and prognostic factors in venous thrombosis. Front Med (Lausanne) 2018;5:147

25 Nicklas JM, Gordon AE, Henke PK. Resolution of deep venous thrombosis: proposed immune paradigms. Int J Mol Sci 2020;21 (06):1-24

26 Borgel D, Bianchini E, Lasne D, Pascreau T, Saller F. Inflammation in deep vein thrombosis: a therapeutic target? Hematology 2019; 24(01):742-750

27 Jezovnik MK, Poredos P. Factors influencing the recanalisation rate of deep venous thrombosis. Int Angiol 2012;31(02):169-175

28 Roumen-Klappe EM, Janssen MCH, Van Rossum J, et al. Inflammation in deep vein thrombosis and the development of postthrombotic syndrome: a prospective study. J Thromb Haemost 2009;7(04):582-587

29 Shbaklo H, Holcroft CA, Kahn SR. Levels of inflammatory markers and the development of the post-thrombotic syndrome. Thromb Haemost 2009;101(03):505-512 
30 Deatrick KB, Elfline M, Baker N, et al. Postthrombotic vein wall remodeling: preliminary observations. J Vasc Surg 2011;53(01): 139-146

31 de Franciscis S, Gallelli L, Amato B, et al. Plasma MMP and TIMP evaluation in patients with deep venous thrombosis: could they have a predictive role in the development of post-thrombotic syndrome? Int Wound J 2016;13(06):1237-1245

32 Henke PK, Varma MR, Moaveni DK, et al. Fibrotic injury after experimental deep vein thrombosis is determined by the mechanism of thrombogenesis. Thromb Haemost 2007;98(05): 1045-1055

33 Betensky M, Amankwah EK, Brandal S, Everett AD, Goldenberg NA. Plasma fibrinolysis, inflammatory markers, and postthrombotic syndrome: preliminary findings from the Kids-DOTT Biobank. Blood Adv 2021;5(01):233-239

34 Holbrook AM, Pereira JA, Labiris R, et al. Systematic overview of warfarin and its drug and food interactions. Arch Intern Med 2005;165(10):1095-1106

35 Popov Aleksandrov A, Mirkov I, Ninkov M, et al. Effects of warfarin on biological processes other than haemostasis: a review. Food Chem Toxicol 2018;113:19-32

36 Rios-Doria J, Favata M, Lasky K, et al. A Potent and selective dual inhibitor of AXL and MERTK possesses both immunomodulatory and tumor-targeted activity. Front Oncol 2020;10:598477

37 Belij S, Miljković D, Popov A, et al. Effects of subacute oral warfarin administration on peripheral blood granulocytes in rats. Food Chem Toxicol 2012;50(05):1499-1507

38 Mirkov I, Popov Aleksandrov A, Demenesku J, et al. Intestinal toxicity of oral warfarin intake in rats. Food Chem Toxicol 2016; 94:11-18

39 Eichbaum FW, Slemer O, Zyngier SB. Anti-inflammatory effect of warfarin and vitamin K1. Naunyn Schmiedebergs Arch Pharmacol 1979;307(02):185-190

40 Yan Y, Ji Y, Su N, et al. Non-anticoagulant effects of low molecular weight heparins in inflammatory disorders: a review. Carbohydr Polym 2017;160:71-81

41 Oduah EI, Linhardt RJ, Sharfstein ST. Heparin: past, present, and future. Pharmaceuticals (Basel) 2016;9(03):9

42 Moaveni DK, Lynch EM, Luke C, et al. Vein wall re-endothelialization after deep vein thrombosis is improved with low-molecularweight heparin. J Vasc Surg 2008;47(03):616-624

43 Downing LJ, Strieter RM, Kadell AM, Wilke CA, Greenfield LJ, Wakefield TW. Low-dose low-molecular-weight heparin is antiinflammatory during venous thrombosis. J Vasc Surg 1998;28 (05):848-854

44 Ceccarelli M, Bani D, Cinci L, et al. Anti-inflammatory effects of low molecular weight heparin derivative in a rat model of carrageenan-induced pleurisy. J Cell Mol Med 2009;13(8B) 2704-2712

45 Bal Dit Sollier C, Drouet L. Non anticoagulant properties of heparin preparations: practical considerations. Sang Thromb. Vaiss. 2013;25:389-398

46 Fareed J, Jeske W, Hoppensteadt D, Clarizio R, Walenga JM. Are the available low-molecular-weight heparin preparations the same? Semin Thromb Hemost 1996;22(Suppl 1):77-91

47 Wang L, Brown JR, Varki A, Esko JD. Heparin's anti-inflammatory effects require glucosamine $6-0$-sulfation and are mediated by blockade of L- and P-selectins. J Clin Invest 2002;110(01): 127-136

48 Ranjbaran H, Wang Y, Manes TD, et al. Heparin displaces interferon$\gamma$-inducible chemokines (IP-10, I-TAC, and Mig) sequestered in the vasculature and inhibits the transendothelial migration and arterial recruitment of T cells. Circulation 2006;114(12):1293-1300

49 Bârzu T, Lormeau J-C, Petitou M, Michelson S, Choay J. Heparinderived oligosaccharides: affinity for acidic fibroblast growth factor and effect on its growth-promoting activity for human endothelial cells. J Cell Physiol 1989;140(03):538-548
50 Deepa PR, Varalakshmi P. Influence of a low-molecular-weight heparin derivative on the nitric oxide levels and apoptotic DNA damage in adriamycin-induced cardiac and renal toxicity. Toxicology 2006;217(2-3):176-183

51 Norrby K. Low-molecular-weight heparins and angiogenesis. APMIS 2006;114(02):79-102

52 Thanaporn P, Myers DD, Wrobleski SK, et al. P-selectin inhibition decreases post-thrombotic vein wall fibrosis in a rat model. Surgery 2003;134(02):365-371

53 Rectenwald JE, Deatrick KB, Sukheepod P, et al. Experimental pulmonary embolism: effects of the thrombus and attenuation of pulmonary artery injury by low-molecular-weight heparin. J Vasc Surg 2006;43(04):800-808

54 Kakkar VV, Hoppenstead DA, Fareed J, et al. Randomized trial of different regimens of heparins and in vivo thrombin generation in acute deep vein thrombosis. Blood 2002;99(06):1965-1970

55 Das SK, Cohen AT, Edmondson RA, Melissari E, Kakkar VV. Lowmolecular-weight heparin versus warfarin for prevention of recurrent venous thromboembolism: a randomized trial. World J Surg 1996;20(05):521-526

56 Gonzalez-Fajardo JA, Arreba E, Castrodeza J, et al. Venographic comparison of subcutaneous low-molecular weight heparin with oral anticoagulant therapy in the long-term treatment of deep venous thrombosis. J Vasc Surg 1999;30(02):283-292

57 Kakkar VV, Gebska M, Kadziola Z, Saba N, Carrasco PBemiparin Investigators. Low-molecular-weight heparin in the acute and long-term treatment of deep vein thrombosis. Thromb Haemost 2003;89(04):674-680

58 López-Beret P, Orgaz A, Fontcuberta J, et al. Low molecular weight heparin versus oral anticoagulants in the long-term treatment of deep venous thrombosis. J Vasc Surg 2001;33(01):77-90

59 Daskalopoulos MEM, Daskalopoulou SS, Tzortzis E, et al. Longterm treatment of deep venous thrombosis with a low molecular weight heparin (tinzaparin): a prospective randomized trial. Eur J Vasc Endovasc Surg 2005;29(06):638-650

60 Romera A, Cairols MA, Vila-Coll R, et al. A randomised open-label trial comparing long-term sub-cutaneous low-molecular-weight heparin compared with oral-anticoagulant therapy in the treatment of deep venous thrombosis. Eur J Vasc Endovasc Surg 2009; 37(03):349-356

61 González-Fajardo JA, Martin-Pedrosa M, Castrodeza J, Tamames S, Vaquero-Puerta C. Effect of the anticoagulant therapy in the incidence of post-thrombotic syndrome and recurrent thromboembolism: comparative study of enoxaparin versus coumarin. J Vasc Surg 2008;48(04):953-959

62 Hull RD, Pineo GF, Brant R, et al; LITE Trial Investigators. Home therapy of venous thrombosis with long-term LMWH versus usual care: patient satisfaction and post-thrombotic syndrome. Am J Med 2009;122(08):762-769

63 Hull RD, Liang J, Townshend G. Long-term low-molecular-weight heparin and the post-thrombotic syndrome: a systematic review. Am J Med 2011;124(08):756-765

64 Guillou S, Beaumont J, Tamareille S, et al. Direct rivaroxabaninduced factor Xa inhibition proves to be cardioprotective in rats. Shock 2020;53(06):730-736

65 Hara T, Fukuda D, Tanaka K, et al. Rivaroxaban, a novel oral anticoagulant, attenuates atherosclerotic plaque progression and destabilization in ApoE-deficient mice. Atherosclerosis 2015;242(02):639-646

66 Blessing E, Zhou Q, Bea F, et al. Evaluation of plaque stability of advanced atherosclerotic lesions in Apo E-deficient mice after treatment with the oral factor Xa inhibitor rivaroxaban. Mediators Inflamm 20112011

67 Laurent M, Joimel U, Varin R, et al. Comparative study of the effect of rivaroxaban and fondaparinux on monocyte's coagulant activity and cytokine release. Exp Hematol Oncol 2014;3 (01):30 
68 Ellinghaus P, Perzborn E, Hauenschild P, et al. Expression of proinflammatory genes in human endothelial cells: comparison of rivaroxaban and dabigatran. Thromb Res 2016;142:44-51

69 Paar V, Jirak P, Gruber S, et al. Influence of dabigatran on proinflammatory cytokines, growth factors and chemokines - slowing the vicious circle of coagulation and inflammation. Life Sci 2020;262:118474

70 Chan MY, Lin M, Lucas J, et al. Plasma proteomics of patients with non-valvular atrial fibrillation on chronic anti-coagulation with warfarin or a direct factor Xa inhibitor. Thromb Haemost 2012; 108(06):1180-1191

71 Prandoni P, Ageno W, Mumoli N, et al. Recanalization rate in patients with proximal vein thrombosis treated with the direct oral anticoagulants. Thromb Res 2017;153:97-100

72 Ferreira T, Huber SC, de Moraes Martinelli B, et al. Low prevalence of post-thrombotic syndrome in patients treated with rivaroxaban. Vasc Pharmacol 2019106608

73 Houghton DE, Lekah A, Macedo TA, et al. Resolution of acute lower extremity deep vein thrombosis with rivaroxaban compared to warfarin. J Thromb Thrombolysis 2020;49(02):199-205

74 Piati PK, Peres AK, de Andrade DO, Jorge MA, Toregeani JF. Analysis of recanalization of deep venous thrombosis: a comparative study of patients treated with warfarin vs. rivaroxaban. J Vasc Bras 2019;18:e20180111

75 de Athayde Soares R, Matielo MF, Brochado Neto FC, Nogueira MP, Almeida RD, Sacilotto R. Comparison of the recanalization rate and postthrombotic syndrome in patients with deep venous thrombosis treated with rivaroxaban or warfarin. Surgery 2019;166(06):1076-1083

76 Prandoni P, Ageno W, Ciammaichella M, et al; DOAC-PTS Investigators. The risk of post-thrombotic syndrome in patients with proximal deep vein thrombosis treated with the direct oral anticoagulants. Intern Emerg Med 2020;15(03):447-452

77 Utne KK, Dahm A, Wik HS, Jelsness-Jørgensen LP, Sandset PM, Ghanima W. Rivaroxaban versus warfarin for the prevention of post-thrombotic syndrome. Thromb Res 2018;163:6-11

78 Jeraj L, Jezovnik MK, Poredos P. Rivaroxaban versus warfarin in the prevention of post-thrombotic syndrome. Thromb Res 2017;157:46-48

79 Cheung YW, Middeldorp S, Prins MH, et al; Einstein PTS Investigators Group. Post-thrombotic syndrome in patients treated with rivaroxaban or enoxaparin/vitamin $\mathrm{K}$ antagonists for acute deep-vein thrombosis. A post-hoc analysis. Thromb Haemost 2016;116(04):733-738

80 Wik H, Sandset P, Eriksson H, et al. Post-thrombotic syndrome in patients with venous thromboembolism treated with dabigatran or warfarin - a long-term cross-sectional follow-up of RE-COVER study patients. J Thromb Haemost 2021;19(10):2495-2503

81 Coleman CI, Beyer-Westendorf J, Bunz TJ, Mahan CE, Spyropoulos AC. Postthrombotic syndrome in patients treated with rivaroxaban or warfarin for venous thromboembolism. Clin Appl Thromb Hemost 2018;24(04):575-582

82 Søgaard M, Nielsen PB, Skjøth F, Kjældgaard JN, Coleman CI, Larsen TB. Rivaroxaban versus warfarin and risk of post-thrombotic syndrome among patients with venous thromboembolism. Am J Med 2018;131(07):787-794

83 Li R, Yuan M, Cheng J, et al. Risk of post-thrombotic syndrome after deep vein thrombosis treated with rivaroxaban versus vitamin-K antagonists: a systematic review and meta-analysis. Thromb Res 2020;196:340-348

84 Camporese G, Bernardi E, Bortoluzzi C, et al. MAC project-monitoring anticoagulant therapy observational study: rationale and protocol. Front Med (Lausanne) 2021;7:584459

85 Salta S, Papageorgiou L, Larsen AK, et al. Comparison of antithrombin-dependent and direct inhibitors of factor Xa or thrombin on the kinetics and qualitative characteristics of blood clots. Res Pract Thromb Haemost 2018;2(04):696-707

86 Baglin T. Prevention of post-thrombotic syndrome: a case for new oral anticoagulant drugs or for heparins? J Thromb Haemost 2012;10(08):1702-1703

87 Nicolaides A, Hull RD, Fareed JCardiovascular Disease Educational and Research Trust European Venous Forum North American Thrombosis Forum International Union of Angiology and Union Internationale du Phlebologie. Prevention of postthrombotic syndrome. Clin Appl Thromb Hemost 2013;19(02):213-215

88 Hull RD, Townshend G. Long-term treatment of deep-vein thrombosis with low-molecular-weight heparin: an update of the evidence. Thromb Haemost 2013;110(01):14-22

89 Rabinovich A, Ducruet T, Kahn SRSOX Trial investigators. Development of a clinical prediction model for the postthrombotic syndrome in a prospective cohort of patients with proximal deep vein thrombosis. J Thromb Haemost 2018;16(02):262-270

90 Amin EE, van Kuijk SMJ, Joore MA, Prandoni P, Ten Cate H, Ten Cate-Hoek AJ. Development and validation of a practical two-step prediction model and clinical risk score for post-thrombotic syndrome. Thromb Haemost 2018;118(07):1242-1249

91 Méan M, Limacher A, Alatri A, Aujesky D, Mazzolai L. Derivation and validation of a prediction model for risk stratification of post-thrombotic syndrome in elderly patients with a first deep vein thrombosis. Thromb Haemost 2018;118(08): 1419-1427 